\title{
Condições para o estudo do comportamento pró-ambiental
}

\author{
Víctor Corral-Verdugo
}

Universidad de Sonora

José Q. Pinheiro

Universidade Federal do Rio Grande do Norte

\begin{abstract}
Resumo
Revisa-se o estado atual da investigação do comportamento pró-ambiental (CPA), assinalando as condições que, segundo os autores, poderiam resultar em obstáculos ou vantagens para essa área de pesquisa. Dentre essas condições, discutem-se o papel desempenhado pela quantidade e qualidade das variáveis preditoras no poder explicativo dos modelos, o problema dos auto-relatos como métodos de mensuração do CPA, o efeito dos contextos de investigação, as influências dos sistemas de análise de dados e a suposta universalidade do CPA.

Palavras-chave: comportamento pró-ambiental, auto-relato, modelos explicativos.
\end{abstract}

Key words: proenvironmental behavior, selfreport, explanatory models.

\begin{abstract}
Conditions for the study of pro-environmental behavior The current status of pro-environmental behavior (CPA) research is reviewed, emphasizing the conditions that, according to the authors, could result in obstacles or advantages to such kind of investigation. Among those conditions we discuss the role played by the quantity and quality of predictive variáveis upon the explanatory power of models, the problem of self-reports as measurement technique of CPA, the effect of research contexts,
\end{abstract}


$\mathrm{D}$ urante as três últimas décadas, o comportamento próambiental (CPA) tem sido um dos objetos de estudo de mai or interesse dentro da Psicologia Ambiental. Graças a esse interesse, tem sido possível descobrir quais características pessoais e quais condições estão associadas a um indivíduo que se mostra responsável em relação ao meio ambiente. Os trabalhos de Borden e Schettino (1979), de Hines, Hungerford e Tomera (1987) e de Schahn e Holzer (1990) mostram que as pessoas mais propensas a cuidar de seu entorno são aquelas com conhecimento, atitudes favoráveis, motivadas, hábeis, com locus de controle interno, responsáveis e com crenças pró-ambientais.

Apesar dos avanços do conhecimento nessa área, aquilo que não sabemos sobre conduta pró-ambiental ainda supera em muito o que sabemos sobre ela. Em estudos típicos do CPA, o erro dos modelos é duas vezes maior do que o seu poder explicativo. Tal fato indica a necessidade de se incorporar variáveis relevantes adicionais àquelas até agora consideradas na predição do CPA. Além disso, alguns investigadores questionam a objetividade e eficácia dos instrumentos de mensuração do CPA e seus determinantes (Cote, 1984; Rathje, 1989). As pesquisas características de comportamento pró-ambiental têm abusado da aplicação quase exclusiva de auto-relatos, o que tem redundado em dados imprecisos e, em muitos casos, contraditórios em relação aos levantados por medidas não-verbais do comportamento. Outro problema consiste nas condições em que se estudam o CPA e seus preditores (Corral, 1997). Tipicamente, uma investigação do CPA é realizada em contextos verbais, colocando os sujeitos investigados fora do cenário ambiental físico-químico que se deseja estudar. Outros aspectos problemáticos são a natureza limitada dos sistemas de análise de dados e a (subentendida) generalização das características pró-ambientais dos indivíduos de países desenvolvidos. Os resultados das pesquisas em países industrializados não podem ser extrapolados de maneira automática para as condições de países mais pobres, os quais devem ser estudados em separado. A análise de todos esses aspectos, então, requer discussão, sendo o propósito do presente trabalho abordá-los, ao menos de maneira sucinta. 


\section{O poder explicativo dos modelos}

Os primeiros estudos acerca do CPA eram, ou de natureza experimental (o que os tornava limitados por necessidade) ou naturalistas (que, em geral, eram limitados por decisão dos pesquisadores). Os behavioristas conduziram a maior parte dos estudos experimentais. Neles se pretendia incrementar a probabilidade de ocorrência de condutas pró-ambientais através de seu reforçamento. Por exemplo, Burgess, Clark e Hendee (1971) entregavam reforçadores positivos aos usuários de um parque público que depositassem seu lixo no lugar apropriado. Os resultados mostraram que a quantidade de lixo depositada fora dos recipientes diminuía visivelmente. Esse procedimento também teve sucesso para incrementar o uso de transporte coletivo. Por exemplo, Everett, Hayward e Meyers (1974) entregavam fichas (tokens) - trocáveis por uma grande variedade de produtos - a todas as pessoas que tomassem um ônibus claramente identificado. A entrega de reforçadores aumentou em $150 \%$ o uso desse tipo de transporte. Outra técnica utilizada consistia no uso de retroalimentação do comportamento, ou seja, dar informação aos sujeitos acerca das consequiências de sua conduta pró- ou antiambiental. Em sua pesquisa, Kohlenberg, Phillips e Proctor (1974), mostraram que, ao oferecer aos sujeitos do estudo retroalimentação contínua sobre seu consumo de energia elétrica, se promovia uma redução desse consumo. Em outros casos visava-se punir a conduta anti-ecológica. Agras, Jacob e Ledebeck (1980) observaram que as multas de consumo excessivo de água produziam uma diminuição significativa no gasto do líquido em consumidores de uma região da Califórnia, durante um período de seca. Esse resultado indicou, segundo aqueles pesquisadores, que o castigo do comportamento antiecológico produz uma redução do mesmo.

Ainda que os resultados tenham se mostrado dramáticos e favoráveis à conservação ambiental, esses delineamentos de pesquisa tinham, ao menos, dois inconvenientes: o número limitado de determinantes (variáveis independentes) do CPA estudado e a dependência extrema dos sujeitos de controles externos ao comportamento, fazendo com que os experimentos fossem de pouca utilidade 
prática. $\mathrm{O}$ número de determinantes do $\mathrm{CPA}$ era limitado, pois os behavioristas estavam mais interessados nos aspectos motivacionais do comportamento pró-ecológico (conseqüências do comportamento) do que em outras variáveis, de tipo disposicional ou situacional, que poderiam afetar o CPA. Ao limitar os estudos a essas variáveis, seus autores também limitavam o poder explicativo dos resultados. $\mathrm{O}$ outro problema relacionava-se ao fato de que os incrementos no comportamento pró-ecológico dependiam da presença do reforçador, ou da conseqüência comportamental. Ao remover essa conseqüência, produzia-se um retorno aos padrões comportamentais da linha de base, o que fazia com que os resultados fossem de pouca utilidade prática.

Se os behavioristas prestavam mais atenção ao controle pelos fatores externos ao comportamento (reforçadores, castigos, antecedentes, ou consequiências da conduta ambiental), os cognitivistas mostravam mais interesse pelas variáveis internas dos indivíduos, que os conduziam ao CPA. Os psicólogos cognitivistas, empregando uma estratégia observacional, concentravam-se na relação entre a conduta ambientalmente responsável e algumas características psicológicas dos indivíduos, como suas atitudes, seus conhecimentos e sua personalidade. Arbuthnot (1977), por exemplo, concluiu que as pessoas que eram política, religiosa e socialmente liberais e que demonstravam um locus interno de controle, eram pessoas que cuidavam bem do ambiente. Weigel (1977) também encontrou uma relação positiva entre ideologia liberal e CPA, enquanto Ostman e Parker (1987) apontaram que possuir conhecimentos acerca do ambiente e seus problemas era um preditor significativo do comportamento próecológico. Outros autores encontraram relações positivas entre possuir atitudes pró-ambientais e o CPA (Hines, Hungerford \& Tomera, 1987) e entre manifestar crenças pró-ambientais e esse comportamento pró-ecológico (Samdahl \& Robertson, 1989). Apesar de convincentes, os resultados eram limitados, conseguindo explicar apenas uma pequena fração do CPA.

Por volta do final da década dos 80 , os estudos experimentais se tornaram mais raros e a pesquisa de tipo correlacional aumentou. 
Uma característica importante dessa mudança foi, não o predomínio da postura cognitivista sobre a behaviorista mas, antes, que os modelos submetidos a análise incluíam variáveis de natureza comportamental e cognitiva. Ao mesmo tempo, incorporaram-se mais variáveis preditivas aos modelos em estudo, fossem eles cognitivistas ou behavioristas. Incluiu-se o estudo de fatores demográficos, o que revelou que os indivíduos jovens são ligeiramente mais responsáveis com o meio do que os mais velhos, os que têm maior renda relatam níveis mais elevados de CPA, e as pessoas com maior nível de instrução tendem a ser mais pró-ecológicas. Essas influências de variáveis demográficas sobre o CPA, no entanto, são praticamente insignificantes (Hines, Hungerford \& Tomera, 1987). Passou-se também a estudar algumas variáveis situacionais, como a pressão social para cuidar do ambiente (Wang \& Katzev, 1990), o efeito de arranjos no planejamento de ambientes, de modo a tornar a conservação do meio mais conveniente para os sujeitos (Katzev, Blake \& Messer, 1993) e o efeito de lembretes (prompts) no comportamento de cuidado dos recursos naturais (Geller, 1985). Todos esses arranjos e condições demonstraram ter influência positiva na proteção ambiental.

Além disso, considerou-se o estudo dos efeitos indiretos dos preditores sobre o CPA como algo relevante e modelos multivariados passaram a ser utilizados, acolhendo de modo mais realista a complexidade das relações entre variáveis. A esse respeito, é necessário mencionar que a maioria dos estudos do CPA até a década dos 80 , só estimava as relações entre a conduta pró-ecológica e seus determinantes de maneira direta, isto é, não se consideravam as noções de variáveis moderadoras ou intermediárias. Por exemplo, considere-se que "A" seja o nível de educação que uma pessoa tenha atingido, "B" seu conhecimento ambiental e "C" o CPA. A educação (A) pode afetar o CPA (C) de maneira direta (AàC), ou fazê-lo de modo indireto, afetando o conhecimento (B, uma variável moderadora), o qual, por sua vez, influi em C (AàBàC). Este tipo de relação tem sido relatado recentemente (Corral, 1996) em estudos que revelam que os efeitos indiretos da educação sobre o CPA podem ser significativos, ainda que as relações diretas entre essa variável demográfica e o cuidado do ambiente não sejam. 
Não obstante o aumento do poder de explicação dos modelos, este é ainda pequeno em comparação com a porção não explicada. Entre as possíveis razões para isso está o fato de que variáveis importantes, tais como fatores situacionais e outras variáveis demográficas além de idade, nível de renda, sexo e grau de escolaridade, ainda estão ausentes dos modelos. Também é preciso considerar o efeito do contexto de investigação sobre as respostas dos sujeitos e o uso de métodos de mensuração compatíveis com o fenômeno em estudo, como trataremos a seguir.

\section{O problema dos auto-relatos}

O auto-relato é um dos métodos mais comuns na investigação de condutas de conservação ambiental. Os questionários e as entrevistas - duas formas de auto-relato - foram utilizados na investigação do consumo racional de produtos (Linn, Vining \& Feeley, 1994), da redução do consumo de energia (Tracy \& Oskamp, 1984), da reutilização (De Young, 1991) e da reciclagem de objetos (Vining \& Ebreo, 1992). Em um estudo típico, pergunta-se aos sujeitos quantas vezes reciclam resíduos de seu lixo, quantas vezes empregam estratégias para economizar água, ou o que fazem para conservar energia elétrica em suas casas. Esses métodos têm a vantagem de proporcionar informação ampla e variada, são fáceis de aplicar e baratos (Bechtel, Marans \& Michelson, 1990). Por outro lado, sempre se supõe que através do auto-relato, os respondentes estarão dando informação confiável sobre seu próprio comportamento.

No entanto, certos autores apontam problemas com o uso de auto-relatos. De acordo com eles, a pressão social para serem vistos como indivíduos responsáveis pode levar as pessoas a superestimar seu próprio comportamento de conservação e a subestimar a quantidade de recursos desperdiçados (Geller, 1981; Warriner, McDougall \& Claxton, 1984). Warriner, McDougall e Claxton (1984) sugerem ainda que a falta de conhecimento e os efeitos do tempo decorrido e da memória podem resultar em auto-relatos incorretos. Ademais, a falta de vontade para responder corretamente pode explicar parcialmente as imprecisões dessas respostas verbais. 
Vários investigadores assinalam que existe uma disparidade entre as medidas de comportamento manifesto, tais como as observações diretas e traços de comportamento (Sommer \& Sommer, 1997), e os relatos verbais da conduta. Ou seja, há uma diferença entre o que as pessoas fazem e o que dizem que fazem. Cote (1984) estudou a correlação entre o relato de consumo de alimentos apresentado por uma amostra de sujeitos e o registro dos restos de sua comida encontrados no lixo. Seus resultados revelaram que só dois produtos, de um total de quinze, produziram correlações maiores do que 0,50. Ao mesmo tempo, McGuire (1984) obteve coeficientes de regressão não-significativos entre os relatos verbais de reciclagem de alumínio e jornais e a presença desses materiais no lixo. Corral, Bernache, Encinas e Garibaldi (1995) replicaram esses resultados com uma amostra maior de produtos e estudando condutas de reutilização, além de reciclagem.

Alguns autores (Corral et al., 1995; Rathje, 1989) sugerem que a falta de correspondência entre os relatos verbais e os registros de comportamento manifesto demonstra o fato de que ambos tipos de métodos medem duas "realidades" diferentes. Os auto-relatos mediriam crenças ou auto-percepção da própria conduta, enquanto os outros métodos registrariam comportamento instrumental. Essa suposição foi corroborada por Corral et al. (1995), em uma investigação em que se produziram dois fatores (ou variáveis latentes): um, a partir dos auto-relatos de condutas de conservação e, o outro, a partir dos restos de produtos potencialmente reutilizáveis e recicláveis, encontrados no lixo. Ao contrastar esses dois fatores, observou-se que eles, com uma correlação insignificante, eram independentes. Em outro estudo (Corral, 1997), constatou-se que os auto-relatos do CPA só se correlacionavam com medidas verbais de variáveis disposicionais do CPA, mas não com medidas não-verbais dessas variáveis, enquanto que as medidas instrumentais (não-verbais) do comportamento pró-ambiental só se relacionavam com os preditores medidos por meios não-verbais.

Do que foi exposto acima, pode-se concluir que os resultados obtidos a partir dos estudos feitos com auto-relatos podem não estar 
indicando a realidade instrumental do CPA, mas apenas os desejos, crenças e convenções dos indivíduos. Se de fato isso se dá, urge complementar a pesquisa feita até agora predominantemente com autorelatos, com outros métodos que levem em conta o aspecto instrumental do comportamento.

\section{O efeito dos contextos de investigação}

Muitos dos estudos que investigam preditores disposicionais da conduta de conservação ambiental, tais como crenças, atitudes e habilidades pró-ambientais, utilizam enunciados verbais para obter respostas verbais relativas a essas disposições e auto-relatos verbais de tais condutas de conservação. O problema é que as respostas dos sujeitos têm pouco a ver com o ambiente estudado (os objetos a conservar e o meio ambiente) e muito mais com os contextos verbais usados pelo investigador. Em tais situações de pesquisa, as respostas dos sujeitos refletem o contexto social criado pelos pesquisadores, e não as características do ambiente supostamente sob estudo (Daniel \& Ittelson, 1981). De fato, para alguns autores (e.g., Evans, 1995), estudar Psicologia Ambiental em ausência do ambiente físico seria, antes, estudar Psicologia Social. Nessas condições, o sujeito não responderia à sua relação com o meio ambiente (supostamente sob investigação), mas sim ao investigador, ou melhor, à relação que se estabelece entre sujeito e pesquisador.

Além disso, é possível que as correlações encontradas entre disposições pró-ambientais medidas verbalmente e as condutas de conservação auto-relatadas sejam explicáveis pela natureza do método de mensuração empregado (relato verbal). O estudo de Corral (1997) parece demonstrar isso, ao indicar que as variáveis preditoras do CPA medidas em contextos verbais só prediziam as respostas verbais dos sujeitos, enquanto que quando essas variáveis eram investigadas em contextos "naturais", quer dizer, o sujeito respondendo frente a estímulos reais, se produzia uma maior correspondência entre essa resposta e o CPA instrumental. Em outro estudo (Corral, 1996), foram obtidas altas correlações entre medidas instrumentais do CPA e o registro dos motivos para conservar o ambiente, registrados na presença de objetos a reutilizar e reciclar. É possível que a alta corres- 
pondência encontrada entre preditores e o CPA seja decorrente de os sujeitos terem sido estudados em um contexto ambiental mais próximo aos estímulos em estudo do que às características sociais de uma entrevista. $\mathrm{O}$ acima exposto sugere, então, a pertinência de se utilizar contextos ambientais para incrementar o poder explicativo dos modelos e, assim, tirar maior proveito da investigação.

\section{Os sistemas de análise de dados}

Uma maneira simples de analisar dados de uma investigação psicoambiental consiste em calcular correlações, no mais simples dos casos, ou uma regressão múltipla, se queremos sofisticar a análise. Muitos dos estudos em CPA têm sido realizados tomando a variável dependente e considerando o efeito único de cada preditor sobre ela. Como resultado, agora sabemos que algumas variáveis, como motivos, habilidades, locus de controle e conhecimento têm um efeito notório sobre o CPA, enquanto que outras variáveis, como renda, nível educacional, idade ou sexo não têm influência significativa, sendo até nula.

Aqueles que lidam com técnicas de regressão múltipla sabem que os efeitos de uma variável $(\mathrm{X})$ sobre a variável dependente $(\mathrm{Y})$ decorrem não somente da relação entre $\mathrm{X}$ e $\mathrm{Y}$, mas também da relação que Y e X têm com outras variáveis. Algumas variáveis, que isoladamente apresentam alta correlação com a variável dependente, podem ter em uma regressão múltipla esse efeito prejudicado pela presença de outras variáveis na equação. Por outro lado, variáveis como a educação, por exemplo, não parecem ter uma influência marcante sobre o CPA, embora exerçam importante influência sobre outras variáveis que, por sua vez, afetam significativamente ao CPA, como é o caso do conhecimento ambiental, já mencionado antes. Isso significa que é necessário considerar influências indiretas, além das influências diretas sobre o CPA, se quisermos ter um quadro mais compreensivo e realista deste tipo de conduta.

Os modelos de regressão múltipla não oferecem a possibilidade de modelar essas relações; o que é possível com a análise de caminho (path analysis), ou com modelos de equações estruturais (Bentler, 
1993). Nesses sistemas de análise é possível estudar efeitos diretos e indiretos de maneira simultânea, assim como incorporar análises fatoriais à análise de caminho, dentro de um mesmo modelo (Bentler, 1993). O uso dos modelos de equações estruturais, que começa paulatinamente a se incorporar ao estudo do CPA, oferece perspectivas muito interessantes para a investigação da conduta ambiental responsável (Corral, 1996; Goldenhar \& Connell, 1993; Taylor \& Todd, 1997).

\section{A suposta universalidade do CPA}

Alguns investigadores europeus e norte-americanos do CPA parecem ter uma visão do mundo segundo a qual o CPA e seus determinantes seriam universais. Essa visão considera que noções como "equilíbrio natural", "pró-ecologia" e "preocupação ambiental" existem em todos os lugares e que sua definição é ao menos semelhante à que eles possuem. Parecem também supor que as características de um sujeito pró-ambientalista no mundo industrializado devem ser as mesmas de um cidadão das nações subdesenvolvidas.

Ainda que todos os seres humanos neste planeta evoluíram contando com um organismo, para fins práticos, idêntico e em cenários parecidos, é indubitável que a diferenciação cultural produziu visões diferentes do entorno. O social, como já vimos, também afeta nossas respostas ao ambiente. $\mathrm{O}$ significado e as implicações de reutilização nos EUA são diferentes dos encontrados no México, por exemplo. $\mathrm{O}$ mesmo se dá com a prática concreta de reciclagem (Corral, 1996). A noção de equilíbrio natural que Dunlap e Van Liere (1978) conceberam em seu "Novo Paradigma Ambiental" soa estranha para ambientalistas do Brasil, assim como para uma amostra de sujeitos brasileiros, conforme relatado por Bechtel, Corral e Pinheiro (1999). Esses autores estudaram as concepções da relação entre seres humanos e natureza, contrastando duas visões: uma, que concebe os seres humanos como parte integrante do meio e, outra, que vê homens e mulheres como senhores da natureza e alheios à ela (Dunlap \& Van Liere, 1978). Bechtel et al. (1999) constataram que os sujeitos estadunidenses possuem uma visão extremadamente dualista das re- 
lações ser humano-ambiente, enquanto que os mexicanos são menos extremados e os brasileiros não mostram nada desse dualismo. A preocupação ambiental dos brasileiros parece ser melhor explicada por concepções não-dicotômicas, que contemplem alguma forma de redefinição de valores presente em uma nova ética de desenvolvimento (Kuhnen, 1995). É o caso, por exemplo, de modelos que levam em conta dimensões ideológicas da preocupação ambiental, como as influências do antropocentrismo, ecocentrismo e apatia (Amérigo, González \& Aragonés, 1995; Thompson \& Barton, 1994). Embora esse modelo de três fatores ainda não seja plenamente satisfatório, parece se adequar muito mais à visão dos brasileiros, oferecendo ainda interessantes sugestões, como a aparente oposição entre ecocentrismo e apatia (Pinheiro, pesquisa em andamento).

Além disso, certos preditores do CPA, como nível de renda, que em estudos norte-americanos e europeus aparecem como promotores da conservação ambiental, aparecem em estudos de países em desenvolvimento como obstáculos para essa conservação (Corral, 1996);

Dada a natureza global dos problemas ambientais, é claro que necessitamos conhecer quais condições em cada região do planeta são responsáveis pelo CPA. Dessa forma, poderemos ter as ferramentas necessárias para contrabalançar os efeitos da degradação ambiental em todo o mundo, e não só em algumas partes dele. É uma pena que os estudos do CPA em países mais pobres estejam reduzidos a um punhado de iniciativas. Devemos, portanto, promover esses esforços e, ao mesmo tempo, convencer as agências de fomento dos países com maior poder econômico que a investigação nessas áreas não é somente interessante, mas também necessária.

\section{À guisa de conclusão}

Revisamos, neste trabalho, algumas condições da pesquisa do comportamento pró-ambiental que, a nosso ver, deveriam estar presentes para otimizar os resultados desse tipo de investigação. Sem pretendermos ser exaustivos, consideramos que uma investigação compreensiva do CPA deveria incluir os aspectos disposicionais (his- 
tóricos) e demográficos dos indivíduos estudados, mas também os fatores situacionais (presentes), com o fim de incrementar o poder explicativo dos modelos. Outro aspecto relevante é considerar medidas do CPA que não se limitem ao uso de auto-relatos e, quando estes forem utilizados, que se leve em conta que os resultados que eles produzem se referem mais a ideais, crenças e valores ligados ao CPA. Não obstante, o aspecto instrumental desse comportamento deveria ser estudado, de preferência empregando outros métodos, como a observação direta ou os traços de comportamento.

Dado que o contexto de investigação é fundamental para os resultados desta área, argumentamos que o CPA deveria ser estudado em presença dos objetos ambientais com os quais os indivíduos entram em contato quando se comportam de maneira responsável (ou irresponsável) com seu ambiente. De outro modo, se estudarmos esse comportamento em contextos verbais como os de uma entrevista clássica, é possível que não estejamos estudando o comportamento próambiental mas, antes, a idéia social que se tem do mesmo.

Um aspecto adicional compreende os sistemas de análise de dados e seus efeitos sobre os resultados de nossos estudos. Com o fim de captar toda a gama de relações entre variáveis que têm algo a ver com a preservação ambiental, é conveniente utilizar modelos causais que estimem efeitos diretos, indiretos, moderados e mediados. Dessa maneira podemos visualizar as influências sobre o CPA que se encontram encobertas, ou que, aparentemente, não existem. Se considerarmos em nossos modelos várias dimensões do mesmo fenômeno (expressas por suas respectivas variáveis), estaremos buscando a solidez da validade de constructo através da abordagem multimétodos (convergência metodológica), recurso fundamental para a concretização da tão propalada interdisciplinaridade.

Por último, ao reconhecer que a cultura tem um peso significativo na história comportamental dos sujeitos, antes de pretender explicar o CPA de uma comunidade, é necessário estudar esse grupo humano, considerando suas idiossincrasias, e o efeito delas sobre a conduta dos indivíduos. Considerando as diferenças culturais, poderemos desenhar um quadro mais realista, compreensivo e de utilidade, que possa nos ajudar, não só a predizer o comportamento ambien- 
tal responsável, mas também a planejar estratégias de intervenção para os crescentes problemas da degradação ambiental.

\section{Referências}

Agras, W. S., Jacob, R. G., \& Ledebeck, M. (1980). The California drought: a quasi-experimental analysis of social policy. Journal of Applied Behavior Analysis, 13, 561-570.

Amérigo, M., González, A., \& Aragonés, J. I. (1995). Antropocentrismo versus ecocentrismo en una muestra de estudiantes. In E. Garrido Martin \& C. Herrero Alonso (Orgs.), Psicología Política, Jurídica y Ambiental (pp. 337-344). Salamanca: Eudema.

Arbuthnot, J. (1977). The role of attitudinal and personality variáveis in the prediction of environmental behavior and knowledge. Environment and Behavior, 9, 217-232.

Bechtel, R., Corral, V., \& Pinheiro, J. Q. (1999). Environmental belief systems: United States, Brazil and Mexico. Journal of Crosscultural Psychology, 30, 122-128.

Bechtel, R., Marans, W., \& Michelson, W. (Orgs.). (1990). Methods in environmental and behavioral research. Malabar: Krieger.

Bentler, P. (1993). EQS, Structural Equations Program Manual. Los Angeles: BMDP Statistical Software.

Borden, R. J., \& Schettino, A. P. (1979). Determinants of environmentally responsible behavior. Journal of Environmental Education, 10, 35-39.

Burgess, R. L., Clark, R. N., \& Hendee, J. C. (1971). An experimental analysis of anti-litter procedures. Journal of Applied Behavior Analysis, 4, 71-75.

Corral, V. (1996). A structural model of reuse and recycling in Mexico. Environment \& Behavior, 28, 665-696.

Corral, V. (1997). Dual "realities" of conservation behavior: selfreports vs. observations of reuse and recycling behavior. Journal of Environmental Psychology, 17, 135-145.

Corral, V., Bernache, G., Encinas, L., \& Garibaldi, L. (1995). A comparison of two measures of reuse and recycling behavior: self-report \& material culture. Journal of Environmental Systems, $23,313-327$.

Cote, J. A. (1984). Use of household refuse analysis to measure usual and period-specific food consumption. American Behavioral Scientist, 28, 71-91. 
Daniel, T. C., e Ittelson, W. H. (1981). Conditions for environmental research: reactions to Ward and Russel. Journal of Experimental Psychology: General, 110, 153-157.

De Young, R. (1991). Some psychological aspects of living lightly: desired lifestyle patterns and conservation behavior. Journal of Environmental Systems, 20, 215-227.

Dunlap, R., \& Van Liere, K. (1978). The "New Environmental Paradigm", a proposed measuring instrument and preliminary results. Journal of Environmental Education, 9, 10-19.

Evans, G. (1995). Measurement of the environment as stressor. Manuscrito não publicado. Cornell University, New York, NY.

Everett, P. B., Hayward, S. C., \& Meyers, A. (1974). The effect of a token reinforcement procedure on bus ridership. Journal of Applied Behavior Analysis, 7, 1-9.

Geller, E. S. (1981). Evaluating energy conservation programs: is verbal report enough? Journal of Consumer Research, 8, 331335.

Geller, E. S. (1985). The behavior change approach to litter management. Journal of Resource Management and Technology, 14, 117-122.

Goldenhar, L. M., \& Connell, C. M. (1993). Understanding and predicting recycling behavior: an application of the theory of the reasoned action. Journal of Environmental Systems, 22, 91-103.

Hines, J. M., Hungerford, H. R., \& Tomera, A. N. (1987). Analysis and synthesis of research on responsible environmental behavior: a meta-analysis. Journal of Environmental Education, 18, 1-8.

Katzev, R., Blake, G., \& Messer, B. (1993). Determinants of participation in multi-family recycling programs. Journal of Applied Social Psychology, 23, 374-385.

Kohlenberg, R., Phillips, J., \& Proctor, W. (1976). A behavioral analysis of peaking in residential electrical-energy consumers. Journal of Applied Behavior Analysis, 9, 13-18.

Kuhnen, A. (1995). Reciclando o cotidiano, representações sociais do lixo. Ilha de Santa Catarina: Letras Contemporaneas.

Linn, N., Vining, J., \& Feeley, P. (1994). Toward a sustainable society: waste minimization through environmentally conscious consuming. Journal of Applied Social Psychology, 24, 1550-1572.

McGuire, R. (1984). Recycling, great expectations and garbage outcomes. American Behavioral Scientist, 28, 41-50.

Ostman, R. E., \& Parker, J. L. (1987). Impact of education, age, newspapers and TV on environmental knowledge, concerns and behaviors. Journal of Environmental Education, 19, 3-9. 
Rathje, W. (1989). The three faces of garbage- measurements, perceptions, behaviors. Journal of Management and Technology, 17, 61-65.

Samdahl, D. M., \& Robertson, R. (1989). Social determinants of environmental concern: Specification and test of the model. Environment \& Behavior, 21, 57-81.

Schahn, J., \& Holzer. E. (1990). Studies of individual environmental concern. Environment and Behavior, 22, 767-786.

Sommer, R., \& Sommer, B. (1997). A practical guide to behavioral research ( $4^{\mathrm{a}}$ ed.). New York: Oxford University Press.

Taylor, S., \& Todd, P. (1997). Understanding the determinants of consumer composting behavior. Journal of Applied Social Psychology, 27, 602-628.

Thompson, S. C. G., \& Barton, M. A. (1994). Ecocentric and anthropocentric attitudes toward the environment. Journal of Environmental Psychology, 14, 149-157.

Tracy, A., \& Oskamp, S. (1984). Relationships among ecologically responsible behaviors. Journal of Environmental Systems, 13, 115-126.

Vining, J., \& Ebreo, A. (1992). Predicting recycling behavior from global and specific environmental attitudes and changes in recycling opportunities. Journal of Applied Social Psychology, $22,1580-1607$.

Wang, T., \& Katzev, R. (1990). Group commitment and resource conservation: Two field experiments on promoting recycling. Journal of Applied Social Psychology, 20, 265-275.

Weigel, R. (1977). Ideological and demographic correlates of proecology behavior. Journal of Social Psychology, 103, 39-47.

Warriner, G. K., McDougall, G. H. C., \& Claxton, J. D. (1984). Any data or none at all? Living with inaccuracies in self-reports of residential energy consumption. Environment \& Behavior, 16, 503-526. 
22 V. Corral-Verdugo e J. Q. Pinheiro

Victor Corral Verdugo, doutor em Psicologia Ambiental pela Universidade do Arizona, Tucson (EUA), é professor do Departamento de Psicología da Universidad de Sonora, México. Endereço para correspondência: Departamento de Psicología, Universidad de Sonora, Rosales e Transversal, Hermosillo, Sonora, México. Telefax (62) 59-21-73. E-mail: vcorral@kunkaak.psicom.uson.mx. José Q. Pinheiro, doutor em Psicologia Ambiental pela Universidade do Arizona, Tucson (EUA), é professor do Departamento de Psicologia da Universidade Federal do Rio Grande do Norte. Endereço para correspondência: Caixa Postal 1507, Natal, RN, CEP 59078-970. Telefax: 084-2153589.

E-mail: pinheiro@cchla.ufrn.br.
Sobre os autores 